PROCEEDINGS OF THE

AMERICAN MATHEMATICAL SOCIETY

Volume 135, Number 6, June 2007, Pages 1851-1859

S 0002-9939(07)08693-5

Article electronically published on January 5, 2007

\title{
UPPER BOUNDS FOR THE VOLUME AND DIAMETER OF $m$-DIMENSIONAL SECTIONS OF CONVEX BODIES
}

\author{
JESÚS BASTERO
}

(Communicated by N. Tomczak-Jaegermann)

\begin{abstract}
In this paper some upper bounds for the volume and diameter of central sections of symmetric convex bodies are obtained in terms of the isotropy constant of the polar body. The main consequence is that every symmetric convex body $K$ in $\mathbb{R}^{n}$ of volume one has a proportional section $K \cap F, \operatorname{dim} F=\lambda n(0<\lambda<1)$, of diameter bounded by

$$
R(K \cap F) \leq \frac{C n^{3 / 4} \log (n+1)}{(1-\lambda)^{3} L_{K^{\circ}}}
$$

whenever the polar body $K^{\circ}$ is in isotropic position $(C>0$ is some absolute constant).
\end{abstract}

\section{Introduction, NOTATION AND RESUlts}

We consider $\mathbb{R}^{n}$ endowed with the canonical euclidian structure. The set $K$ will be a convex body in $\mathbb{R}^{n}$, i.e., a compact convex set in $\mathbb{R}^{n}$ having a nonempty interior. We shall say that $K$ is in isotropic position if its centroid is the origin

$$
\int_{K} x d x=0
$$

and there is a constant $\alpha>0$ such that

$$
\int_{K}\langle x, \theta\rangle^{2} d x=\alpha
$$

for all $\theta \in S^{n-1}$. Every convex body in $\mathbb{R}^{n}$ has a unique, up to orthogonal transformation, isotropic position (by a position of a convex body here we mean any affine transformation of the set, with determinant equal to 1 ). We recall the definition of the isotropy constant $L_{K}$ of a convex body $K$

$$
n L_{K}^{2}|K|^{2 / n}=\inf _{\substack{S \in S L(n) \\ t \in \mathbb{R}^{n}}} \frac{1}{|K|} \int_{K}|t+S x|^{2} d x,
$$

Received by the editors September 14, 2005 and, in revised form, February 8, 2006 and February 14, 2006.

2000 Mathematics Subject Classification. Primary 46B20, 52A20, 52A40.

Key words and phrases. Asymptotic geometric analysis, diameter of sections.

The author was partially supported by DGA (Spain), MCYT (Spain) MTM2004-03036 and EU Project MRTN-CT-2004-511953 and is grateful to the Erwin Schrödinger Institut in Wien, where part of this work was carried out. 
so, if $K$ is in in isotropic position and $|K|=1$,

$$
L_{K}^{2}=\int_{K}\langle x, \theta\rangle^{2} d x
$$

for all $\theta \in S^{n-1}$.

In the sequel we will assume that $|K|=1$. It is a major problem in asymptotic convex geometry to prove that the isotropy constant of any convex body $K$ in $\mathbb{R}^{n}, L_{K}$, is bounded from above by an absolute constant (independent even on the dimension). It is well known that this fact is equivalent to the slicing problem: there exists an absolute constant $c>0$ with the following property: if $K$ is a convex body in $\mathbb{R}^{n}$ there exists a hyperplane $H$ such that $|K \cap H|_{n-1}>c$. The best known bound for the isotropy constant was given by Bourgain $L_{K} \leq C n^{1 / 4} \log n$ (see [Bo1, Bo2 and also D and Pa1 for the nonsymmetric case). Very recently, using a new concentration inequality of G. Paouris ([Pa2, $\mathrm{Pa}])$, B. Klartag has just improved Bourgain's estimate proving that $L_{K} \leq C n^{1 / 4}$ (see [K12]).

Let us assume that the convex body $K$ of volume one has its centroid in the origin. The circumradius $R(K)$ is the quantity

$$
R(K)=\max \{|x| ; x \in K\}=\max \left\{1 /\|\theta\|_{K} ; \theta \in S^{n-1}\right\} .
$$

A simple computation shows that $C \sqrt{n} \leq R(K)$. Moreover if $K$ is isotropic $R(K) \leq \sqrt{n(n+2)} L_{K}$ (cf. [KLS] ) and if instead of that, $K^{\circ}$ is in isotropic position, then

$$
R(K) \leq \frac{C n}{L_{K^{\circ}}} \leq C n,
$$

where $C$ is an absolute constant (see item 3 in the Remarks below).

In this paper some upper bounds for the volume and circumradius of sections of symmetric convex bodies with volume one are obtained in terms of the isotropy constant of the polar body.

The proof we use in proving the part ii) of Theorem 1.1 is based in the methods appearing in the paper Bo2 in which Bourgain obtained some sharp estimates for isotropic $\psi_{2}$-convex bodies. Our result is equivalent to that appearing in Bo2 (lemma on page 116), at least for high-dimensional sections. However, the approach we use here presents some differences. We use the $M$-ellipsoid associated to every convex body and sharp estimates on covering numbers given by Pisier (see [Pi]) instead of using the $\ell$-ellipsoid and Talagrand majoration theorem.

The main result we achieve is the following.

Theorem 1.1. Let $K$ be a centrally symmetric convex body in $\mathbb{R}^{n}$ with volume $|K|=1$ such that $K^{\circ}$ is in isotropic position. Let $E$ be any $m$-dimensional subspace, $1 \leq m \leq n$. Then

i)

$$
|K \cap E|_{m}^{1 / m} \leq \frac{C n}{m^{3 / 4} L_{K^{\circ}}},
$$

ii) if $0<\delta<1 / 2$ there exists another subspace $F \subset E,(1-2 \delta) m \leq \operatorname{dim} F \leq$ $(1-\delta) m$ such that

$$
R(K \cap F) \leq \frac{C}{\delta^{3}} \frac{\log (m+1) n^{5 / 4}}{m^{1 / 2} L_{K^{\circ}}}
$$

for some absolute constant $C>0$ (independent of $K$ and of the dimensions $m, n$ ). 
Remarks. 1. The bound (1.2) for the high dimensions gives the better known bound for the isotropy constant. In fact, it is clear for $m=n$ simply by taking $E=\mathbb{R}^{n}$. A similar situation happens for $m=n(1-f(n))$ whenever $f(n)=O(1 / \log n)$. Indeed, as a consequence of Hensley inequalities (cf. [H] ) or Ball's result (cf. [B2], Lemma 6) we know that

$$
L_{K}|E \cap K|_{m}^{1 /(n-m)} \geq C
$$

whenever that $K$ is a symmetric convex body in $\mathbb{R}^{n}$ of $|K|=1$ in isotropic position, $E$ is any $m$-dimensional subspace of $\mathbb{R}^{n}$ and $C>0$ is a numerical constant. Then if the convex body $K$ is not assumed to be in isotropic position the inequality before is true at least for one $m$-dimensional subspace $E$ and so, taking into account (1.2) we have

$$
\left(\frac{C_{1}}{L_{K}}\right)^{(n-m) / m} \leq|K \cap E|_{m}^{1 / m} \leq \frac{C_{2} n}{m^{3 / 4} L_{K^{\circ}}},
$$

and by using the rough estimates for the isotropy constant (for instance, $L_{K} \leq$ $C \sqrt{n}$ ), we would have

$$
\left(\frac{C_{1}}{\sqrt{n}}\right)^{(n-m) / m} \leq \frac{C_{2} n}{m^{3 / 4} L_{K^{\circ}}}
$$

When $m=n(1-f(n))$ the inequality gives the better known estimate for the isotropy constant.

2. Apart from random estimates obtained recently by different authors (see, for instance, Giannopoulos and Milman in GM1, GM2 and GM3, Giannopoulos, Milman and Tsolomitis in GMT, Litvak and Tomczak-Jaegermann in [LT] and Klartag in [K11]) the most recent result in this line is that by Bourgain, Klartag and Milman (see $[\mathrm{BKM}]$ ) who proved that if $K_{n}$ is an isotropic convex body of volume one such that $L_{K_{n}}$ is the worst possible constant in dimension $n$, then for any subspace $F$ of dimension $n-m,\left|K_{n} \cap F\right|_{n-m}^{1 / m} \leq C$, for some absolute constant $C$. In our situation, if $K$ is a symmetric convex body such that $L_{K^{0}}=L_{K_{n}}$, then by using the fact that $L_{m} \leq C L_{n}$, we have that $|K \cap E|_{m}^{1 / m} \leq C \frac{n}{m}$.

3. It is clear that no upper bounds for the circumradius of a general symmetric convex body can exist without fixing a position. However if $K^{\circ}$ is in isotropic position, then the expression (1.2) for $m=1$ gives $R(K) \leq C n$. We can also give a shorter proof using the reverse Blaschke-Santaló's inequality (see $\mathrm{BM}$ ). Indeed, since the convex body $K^{\circ}$ is in isotropic position and its volume is $\left|K^{\circ}\right|_{n}^{1 / n} \sim 1 / n$, the isotropic constant satisfies

$$
L_{K^{\circ}}^{2} \sim \frac{n}{\left|K^{\circ}\right|_{n}} \int_{K^{\circ}}|x|^{2} d x
$$

according to (1.1). Then we have

$$
L_{K^{\circ}}^{2} \leq C \frac{n^{2}}{\left|K^{\circ}\right|} \int_{K^{\circ}}\langle x, \theta\rangle^{2} d x \leq C n^{2}\|\theta\|_{K}^{2} .
$$

$\left(\|\cdot\|_{K}\right.$ represents the norm in $\mathbb{R}^{n}$ whose unit ball is $K$.)

If $K$ is a general convex body in $\mathbb{R}^{n}$ with its centroid in the origin, using $K \subset$ $K-K$ and Rogers-Shephard's inequality there exists a position of $K$, say $\tilde{K}$, for which

$$
R(\tilde{K}) \leq C n
$$

4. We can improve the above estimate on the circumradius only for highdimensional sections of convex bodies of volume one, whose polars are in isotropic 
position. For instance, given $0<\lambda<1$, by taking $m=n$ and $\delta=(1-\lambda) / 2$ in Theorem 1.1, we obtain that for any convex body $K$ satisfying this hypothesis there exists some $\lambda n$-dimensional section for a subspace $F$ such that

$$
R(K \cap F) \leq \frac{C n^{3 / 4} \log (n+1)}{(1-\lambda)^{3} L_{K^{\circ}}} .
$$

Taking polars and renormalizating volumes we can express this result in terms of the polar body in the following way: If $K$ is an isotropic symmetric convex body in $\mathbb{R}^{n}$ with volume one, then given $0<\lambda<1$ there exists some orthogonal projection on the subspace $F$ of $\mathbb{R}^{n}$ such that

$$
P_{F}(K) \supseteq C(1-\lambda)^{3} \frac{L_{K} n^{1 / 4}}{\log (1+n)} D_{\lambda n}
$$

where $D_{\lambda n}$ denotes the Euclidean unit ball in dimension $\lambda n$.

Let us eventually introduce some notation. We represent $\ell_{2}^{n}$ the normed space $\mathbb{R}^{n}$ with the Euclidean norm. Note that $|\cdot|$ will represent the Euclidian norm and also the volume $|K|=|K|_{n}$ (if we want to emphasise the dimension). By $C, c$ we will represent absolute constants which can vary from line to line. The expression $\sim$ denotes equivalence of two quantities up to an absolute factor.

\section{Proof of the theorem}

Proof. i) The case $m=n$ is just the estimate for the isotropy constant. We only consider $1 \leq m \leq n-1$. By using Bourgain-Milman's reverse Blaschke-Santaló's inequality (see $[\overline{B M}]$ ) we have that

$$
\begin{aligned}
|K \cap E|_{m}^{1 / m} & \leq \frac{C}{m\left|(K \cap E)^{\circ}\right|_{m}^{1 / m}} \\
& =\frac{C}{m\left|P_{E}\left(K^{\circ}\right)\right|_{m}^{1 / m}}
\end{aligned}
$$

and also using Fubini's theorem and Brunn-Minkowski inequality

$$
\left|K^{\circ}\right|_{n}^{1 / n} \leq\left|K^{\circ} \cap E^{\perp}\right|_{n-m}^{1 / n}\left|P_{E}\left(K^{\circ}\right)\right|_{m}^{1 / n} .
$$

Then

$$
|K \cap E|_{m}^{1 / m} \leq \frac{C}{m\left|K^{\circ}\right|^{1 / m}}\left|K^{\circ} \cap E^{\perp}\right|_{n-m}^{1 / m} .
$$

Now we use a fact which can be deduced from a well-known result by Milman-Pajor (cf. MP, Proposition 3.11) (this result can be found implicit in K. Ball (B1])): if $T$ in $\mathbb{R}^{n}$ is an isotropic symmetric convex body, then

$$
\left|T \cap E^{\perp}\right|_{n-m}^{1 / m} \leq C \frac{L_{m}}{L_{T}}|T|_{n}^{(n-m) / n m},
$$

where $C$ is an absolute constant and $L_{m}$ is the supremun of the isotropy constants of all $m$-dimensional convex bodies. Hence, we apply this result to $T=K^{\circ}$, taking into account that $\left|K^{\circ}\right|_{n}^{1 / n} \sim C / n$ by reverse Blaschke-Santaló's inequality. Eventually the better known estimate for the isotropy constant gives the result. 
ii) Since the convex body $K^{\circ}$ is in isotropic position and its volume is $\left|K^{\circ}\right|{ }_{n}^{1 / n} \sim$ $1 / n$, the isotropic constant $L=L_{K^{\circ}}$ satisfies

$$
L^{2} \sim \frac{n}{\left|K^{\circ}\right|_{n}} \int_{K^{\circ}}|x|^{2} d x
$$

according to (1.1).

Let $E$ be any $m$-dimensional subspace of $\mathbb{R}^{n}$. We denote by $X$ the $m$-dimensional normed space $X=\left(E,\|\cdot\|_{K \cap E}\right)$, where $\|\cdot\|_{K \cap E}$ is the norm on $E$ whose unit ball is $K \cap E$. We denote by $B$ the polar set of $K \cap E$ in $E$ so the dual space is $X^{*}=\left(E,\|\cdot\|_{B}\right)$, and it is well known that $B=P_{E}\left(K^{\circ}\right)$, where $P_{E}$ denotes the orthogonal projection on $E$.

We consider the $M$-ellipsoid associated to the symmetric convex body (in $E$ ), $K \cap E$ (see [Pi]), i.e. given any $0<p<2$ there exists an isomorphism $u: \ell_{2}^{m} \rightarrow X$ such that

$$
\max \left\{d_{k}(u), c_{k}\left(u^{-1}\right), e_{k}(u), e_{k}\left(u^{*}\right), e_{k}\left(u^{-1}\right), e_{k}\left(u^{-1 *}\right)\right\} \leq \frac{C}{\sqrt{2-p}}\left(\frac{m}{k}\right)^{1 / p}
$$

for some absolute constant $C>0$ and for all $k=1, \ldots, m$, where the $d_{k}, c_{k}, e_{k}$ 's are the corresponding Kolmogorov, Gelfand and entropy numbers. Furthermore

$$
\begin{aligned}
\max & \left\{\log N\left(u\left(D_{m}\right), t K \cap E\right), \log N\left(u^{*}(B), t D_{m}\right),\right. \\
& \left.\log N\left(K \cap E, t u\left(D_{m}\right)\right), \log N\left(D_{m}, t u^{*}(B)\right)\right\} \\
& \leq\left(\frac{C}{\sqrt{2-p}}\right)^{p} \frac{m}{t^{p}}
\end{aligned}
$$

for all $t \geq C / \sqrt{2-p}$, where $C>0$ is an absolute constant (see, for instance, [Pi], Theorem 7.13 and Corollaries 7.15 and 7.16).

We can assume that the isomorphism $u$ has a diagonal expression if we take the canonical basis $\left\{\varepsilon_{i}\right\}_{i=1}^{m}$ in $\ell_{2}^{m}$ and some orthogonal basis $\left\{e_{i}\right\}_{i=1}^{m}$ (with respect to the euclidian structure of $\mathbb{R}^{n}$ ) in $E$ such that $u\left(\varepsilon_{i}\right)=\lambda_{i} e_{i}$, for some $\lambda_{i}>0$, $1 \leq i \leq m$ in such a way that $\lambda_{1} \leq \lambda_{2} \leq \cdots \leq \lambda_{m}$. Let $1 \leq k<m$. Since

$$
c_{k}\left(u^{-1}\right) \leq \frac{C}{\sqrt{2-p}}\left(\frac{m}{k}\right)^{1 / p}
$$

(see (2.5)), by definition of the Gelfand numbers, there exists a subspace $F_{1} \subseteq E$ with $\operatorname{dim} F_{1}>m-k$, such that

$$
\left|u^{-1}(x)\right| \leq \frac{C}{\sqrt{2-p}}\left(\frac{m}{k}\right)^{1 / p}\|x\|_{K}
$$

for all $x \in F_{1}$. Let $F_{2}=\operatorname{span}\left\{e_{1}, \ldots, e_{m-k}\right\}$. It is clear that $F=F_{1} \cap F_{2}$ verifies that $m-2 k<\operatorname{dim} F \leq m-k$ and

$$
\left|u^{-1}(x)\right|=\left|\sum_{i=1}^{m-k} \lambda_{i}^{-1} x_{i} \varepsilon_{i}\right| \geq \frac{1}{\lambda_{m-k}}\left(\sum_{i=1}^{m-k} x_{i}^{2}\right)^{1 / 2}=\frac{|x|}{\lambda_{m-k}}
$$


for all $x=\sum_{i=1}^{m-k} x_{i} e_{i} \in F$. So,

$$
\begin{aligned}
|x| & \leq \lambda_{m-k} \frac{C}{\sqrt{2-p}}\left(\frac{m}{k}\right)^{1 / p}\|x\|_{K} \\
& \leq \frac{1}{k}\left(\sum_{i=m-k+1}^{m} \lambda_{i}\right) \frac{C}{\sqrt{2-p}}\left(\frac{m}{k}\right)^{1 / p}\|x\|_{K} \\
& \leq \frac{1}{k}\left(\sum_{i=1}^{m} \lambda_{i}\right) \frac{C}{\sqrt{2-p}}\left(\frac{m}{k}\right)^{1 / p}\|x\|_{K}
\end{aligned}
$$

for all $x \in F$. We will assume the following.

\section{Claim.}

$$
\sum_{i=1}^{m} \lambda_{i} \leq \frac{C n^{(3 / 2-1 / 2 p)} m^{1 / p}}{L \sqrt{2-p}}
$$

We first finish the proof. If we assume that $k=\delta m$, for some $0<\delta<1 / 2$, then

$$
|x| \leq \frac{C}{(2-p) \delta^{1+1 / p}} \frac{n^{3 / 2-1 / 2 p}}{L m^{1-1 / p}}
$$

for all $x \in F \cap K$, and we achieve the result, by taking $p=2-1 / \log m$.

Proof of the Claim. We consider $\ell_{2}^{m}$ canonically embedded in $\ell_{2}^{n}$, and we denote by $\left\{\varepsilon_{i}\right\}_{i=1}^{n}$ its canonical basis; in the same way, we extend the orthogonal basis $\left\{e_{i}\right\}_{i=1}^{m}$ from $E$ to all $\mathbb{R}^{n}$. Let $P_{E}: \mathbb{R}^{n} \rightarrow E$ be the orthogonal projection on $E$.

Let $K_{1} \subseteq \ell_{2}^{n}$ be the convex set defined by

$$
K_{1}=\left\{x=\sum_{i=1}^{n} x_{i} \varepsilon_{i} \in \ell_{2}^{n} ; \quad \sum_{i=1}^{n} x_{i} e_{i} \in K^{\circ}\right\} .
$$

$K_{1}$ is an orthogonal copy of $K^{\circ}$, therefore they have the same volume and isotropy constant, and hence, by (2.4),

$$
\frac{C}{n^{2}} L^{2} \leq \frac{1}{\left|K_{1}\right|_{n}} \int_{K_{1}} x_{i}^{2} d x
$$

for an absolute constant and for all $1 \leq i \leq n$. Hence

$$
\begin{aligned}
\frac{C}{n^{2}} L^{2} \sum_{i=1}^{m} \lambda_{i} & \leq \frac{1}{\left|K_{1}\right|_{n}} \int_{K_{1}} \sum_{i=1}^{m} \lambda_{i} x_{i}^{2} d x \\
& =\frac{1}{\left|K_{1}\right|_{n}} \int_{K_{1}}\left\langle\sum_{i=1}^{n} x_{i} \varepsilon_{i}, u^{*}\left(\sum_{i=1}^{m} x_{i} e_{i}\right)\right\rangle d x \\
& =\frac{1}{\left|K_{1}\right|_{n}} \int_{K_{1}}\left\langle x, u^{*} \circ P_{E}\left(\sum_{i=1}^{n} x_{i} e_{i}\right)\right\rangle d x \\
& \leq \frac{1}{\left|K_{1}\right|_{n}} \int_{K_{1}} \max _{z \in u^{*}(B)}\langle x, z\rangle d x
\end{aligned}
$$

since $B$, which is the polar of $K \cap E$ in $E$, can be expressed as $B=P_{E}\left(K^{\circ}\right)$ and $u^{*}:\left(E,\|\cdot\|_{B}\right) \rightarrow \ell_{2}^{m}$. 
Now we follow the method appearing in [D, Gi], Pa1] in order to estimate from above the last integral. If we assume that $u^{*}(B) \subset R D_{m}$, for some $R>0$ large enough, we use the Dudley-Fernique's technique. Let $N \in \mathbb{N}$ (to be chosen later). By using (2.8), it is clear that for every $j=1, \ldots, N$ there exist points $y_{1}^{(j)}, \ldots, y_{N_{j}}^{(j)}$ such that

$$
u^{*}(B) \subset \bigcup_{i=1}^{N_{j}}\left(y_{i}^{(j)}+\frac{R}{2^{j}} D_{m}\right)
$$

where

$$
\log N_{j} \leq\left(\frac{C}{\sqrt{2-p}}\right)^{p} \frac{m 2^{j p}}{R^{p}} .
$$

Therefore, for every $z \in u^{*}(B)$ and $1 \leq j \leq N$ we choose the points $y^{(j)}$ such that $\left|z-y^{(j)}\right| \leq R / 2^{j}$. Hence

$$
\begin{aligned}
z & =0+\left(y^{(1)}-0\right)+\left(y^{(2)}-y^{(1)}\right)+\cdots+\left(y^{(N)}-y^{(N-1)}\right)+\left(z-y^{(N)}\right) \\
& =w_{1}+\cdots+w_{N}+w
\end{aligned}
$$

where

$$
\begin{aligned}
& \left|w_{j}\right| \leq\left|y^{(j)}-z\right|+\left|z-y^{(j-1)}\right| \leq \frac{3 R}{2^{j}}, \\
& \left.|w|=\mid z-y^{(N)}\right) \mid \leq \frac{R}{2^{N}} .
\end{aligned}
$$

Each vector $w_{j}$ belongs to a finite set $\mathcal{F}_{j}$ of cardinality $\left|\mathcal{F}_{j}\right| \leq N_{j} N_{j-1}$, so

$$
\log \left|\mathcal{F}_{j}\right| \leq 2\left(\frac{C}{\sqrt{2-p}}\right)^{p} \frac{m 2^{j p}}{R^{p}} .
$$

Therefore

$$
\int_{K_{1}} \max _{z \in u^{*}(B)}\langle x, z\rangle d x \leq \sum_{j=1}^{N} \int_{K_{1}} \max _{w_{j} \in \mathcal{F}_{j}}\left\langle x, w_{j}\right\rangle d x+\int_{K_{1}} \max _{w \in \frac{R}{2^{N}} D_{m}}\langle x, w\rangle d x .
$$

Since $K_{1}$ is in isotropic position, the last summand verifies

$$
\begin{aligned}
\frac{1}{\left|K_{1}\right|_{n}} \int_{K_{1}} \max _{w \in \frac{R}{2^{N} D_{m}}}\langle x, w\rangle d x & \leq \frac{R}{2^{N}} \frac{1}{\left|K_{1}\right|_{n}} \int_{K_{1}}|x| d x \\
& \leq \frac{R}{2^{N}}\left(\frac{1}{\left|K_{1}\right|_{n}} \int_{K_{1}}|x|^{2} d x\right)^{1 / 2} \\
& \leq \text { by (2.4) } \leq \frac{C R}{2^{N}} \frac{L}{\sqrt{n}} .
\end{aligned}
$$

We use the following well-known fact (which is a consequence of Borell's lemma; see [MS]): if $\tilde{K}$ is an isotropic convex body with volume $|\tilde{K}|_{n}=1$, then

$$
\int_{\tilde{K}} \max _{i=1}^{N}\left|\left\langle x, y_{i}\right\rangle\right| d x \leq C L_{\tilde{K}} \log N \max _{i=1}^{N}\left|y_{i}\right|
$$


for any finite family of unit vectors $\left\{y_{1}, \ldots, y_{N}\right\}$, where $C>0$ is an absolute constant. Then we get that

$$
\begin{aligned}
\frac{n}{\left|K_{1}\right|_{n}} \int_{K_{1}} \max _{w_{j} \in \mathcal{F}_{j}}\left|\left\langle x, w_{j}\right\rangle\right| d x & \leq C \frac{\left|K_{1}\right|_{n}^{-1 / n}}{\left|K_{1}\right|_{n}} \int_{K_{1}} \max _{w_{j} \in \mathcal{F}_{j}}\left|\left\langle x, w_{j}\right\rangle\right| d x \\
& \leq C_{1} L \log \left|\mathcal{F}_{j}\right| \max _{w_{j} \in \mathcal{F}_{j}}\left|w_{j}\right| \\
& \leq C_{1} L\left(2\left(\frac{C}{\sqrt{2-p}}\right)^{p} \frac{m 2^{j p}}{R^{p}}\right) \frac{3 R}{2^{j}} \\
& \leq \frac{C C_{1} L m}{(2-p)^{p / 2}} \frac{2^{j(p-1)}}{R^{p-1}} .
\end{aligned}
$$

Taking into account (2.12) and (2.13) we have

$$
\begin{aligned}
L \sum_{i=1}^{m} \lambda_{i} & \leq C n\left(\frac{R}{2^{N}} \sqrt{n}+\frac{C C_{1} m}{(2-p)^{p / 2}} \sum_{j=1}^{N} \frac{2^{j(p-1)}}{R^{p-1}}\right) \\
& \leq C n\left(\frac{R}{2^{N}} \sqrt{n}+\frac{C C_{1} m}{(2-p)^{p / 2}(p-1)}\left(\frac{2^{N}}{R}\right)^{p-1}\right) .
\end{aligned}
$$

We optimize by taking

$$
\frac{R}{2^{N}}=\left(\frac{C C_{1} m}{\sqrt{n}(2-p)^{p / 2}}\right)^{1 / p}
$$

and hence the claim holds.

\section{ACKNOWLEDGMENT}

The author is very indebted to Bo'az Klartag for permitting the inclusion of this version and proof of part i) of the theorem, which very much improved an earlier version.

\section{REFERENCES}

[B1] K. BALL, Isometric problems in $\ell^{p}$ and sections of convex bodies, $\mathrm{Ph} . \mathrm{D}$. Thesis, Cambridge University (1986).

[B2] K. BALL, Logarithmically concave functions and sections of convex sets in $\mathbb{R}^{n}$, Studia Math. 88 (1988), pp. 69-84. MR0932007(89e:52002)

[BKM] J. Bourgain, B. Klartag and V. Milman, Symmetrization and isotropic constants of convex bodies, Geometric Aspects of Functional Analysis, Lecture Notes in Math. 1850, Springer (2004), 101-116. MR.2087154 (2005i:52005)

[Bo1] J. Bourgain, On the distribution of polynomials on high dimensional convex sets, Lecture Notes in Math. Springer, 1469 (1991), 127-137. MR.1122617 (92j:52007)

[Bo2] J. Bourgain, On the isotropy-Constant Problem for "PSI-2"-Bodies, Lecture Notes in Math. Springer, 1807 (2003), 114-121. MR2083391 (2006c:46011)

[BM] J. Bourgain and V. Milman, New volume ratio properties for convex symmetric bodies in $\mathbb{R}^{n}$, Invent. Math. 88 (1987), pp. 319-340. MR0880954 (88f:52013)

[D] S. DAR, Remarks on Bourgain's problem on slicing convex bodies, in Geom. Aspects of Funct. Analysis (Lindenstrauss-Milman eds.), Operator Theory: Advances and Applications 77 (1995), 61-66. MR1353449 (96j:46010)

[Gi] A. Giannopoulos, Notes on isotropic convex bodies, http://itia.math.uch.gr/ apostolo/notes.html.

[GM1] A. Giannopoulos And V.D. Milman, On the diameter of proportional sections of a symmetric convex body, International Mathematical Research Notices 1 (1997), pp. 5-19. MR:1426731(97k:52003) 
[GM2] A. Giannopoulos And V.D. Milman, How small can the intersection of a few rotations of a symmetric convex body be?, C. R. A. S. Paris 325 (1997), pp. 389-393. MR.1467092 (98e:52003)

[GM3] A. Giannopoulos And V.D. Milman, Mean width and diameter of proportional sections of a symmetric convex body, J. Reine Angew. Math. 497 (1998), pp. 113-139. MR1617429 (99c:52006)

[GMT] A.Giannopoulos, V.D. Milman and A. Tsolomitis, Asymptotic formulas for the diameter of sections of symmetric convex bodies, Journal of Functional Analysis 223 (1), (2005), pp. 86-108. MR2139881 (2006b:46010)

$[\mathrm{H}]$ D. Hensley, Slicing convex bodies - bounds of slice area in terms of the body's covariance, Proc. Amer. Math. Soc. 79 (1980), pp. 619-625. MR0572315 (81j:52008)

[KLS] R. Kannan, L. Lovasz And M. Simonovits, Isoperimetric problems for convex bodies, Discrete Comput. Geom. 13 (1995), pp. 541-559. MR.1318794 (96e:52018)

[Kl1] B. Klartag, A geometric inequality and a low M-estimate, Proc. Amer. Math. Soc. 132 (9) (2004), pp. 2919-2628. MR2054787 (2004m:46029)

[Kl2] B. Klartag, On convex perturbations with a bounded isotropic constant, to appear in Geom. and Funct. Anal.

[LT] A. Litvak and N. Tomczak-Jaegermann, Random aspects of high dimensional convex bodies. In Geometric Aspects of Functional Analysis, Israeli Seminar, Lecture Notes in Math., 1795, Springer, 2000, pp. 169-191. MR1796719 (2002b:52005)

[MP] V. Milman AND A. PAJOR, Isotropic positions and inertia ellipsoids and zonoids of the unit ball of a normed n-dimensional space, GAFA Seminar 87-89, Springer Lecture Notes in Math., 1376 (1989), pp. 64-104. MR1008717 (90g:52003)

[MS] V. Milman and G. Schechtman, Asymptotic theory of finite dimensional normed spaces, Lecture Notes in Math. 1200 (1986). MR0856576 (87m:46038)

[Pa1] G. PAOURIS, On the isotropic constant of non-symmetric convex bodies, Lecture Notes in Math., Springer, 1745 (2000), 239-243. MR1796722(2002b:52013)

[Pa2] G. PaOuris, Concentration of mass on isotropic convex bodies, C. R. Acad. Sci. Paris., Ser. I 342, (2006), 179-182. MR2198189

[Pa3] G. Paouris, Concentration of mass in convex bodies, to appear in Geom. Funct. Anal.

[Pi] G. PIsIER, The Volume of Convex bodies and Banach Space Geometry, Cambridge Tracts in Mathematics 94 (1989). MR1036275 (91d:52005)

Departamento de Matemáticas, Facultad de Ciencias, Universidad de Zaragoza, 50009 ZARAGOZA, SPAIN

E-mail address: bastero@unizar.es 